\title{
Modelación del parámetro de identificación de diagnóstico PID's, del sensor de temperatura de refrigerante del motor ECT del sistema de control de inyección electrónica de combustible EFI, mediante regresión no lineal
}

\section{Modeling of the diagnostic identification parameter PID's, of the ECT engine coolant temperature sensor of the electronic fuel injection control system EFI, by means of non-linear regression}

\author{
Washington German Erazo \\ Jose Lizandro Quiroz \\ Byron Joel Salazar \\ Alex Dario Pallo \\ Leonidas Antonio Quiroz Erazo \\ Victor Danilo Zambrano León \\ Universidad de las Fuerzas Armadas-ESPE, Ecuador
}

Autor de correspondencia: wgerazo@espe.edu.ec,jlquiroz@espe.edu.ec, bjsalazar@espe.edu.ec. adpallo@espe.edu.ec, laquiroz@espe.edu.ec, vdzambrano@espe.edu.ec

Fecha de recepción: 02 de julio de 2017 - Fecha de aceptación: 30 de noviembre de 2017

Resúmen: A través de la experimentación en esta investigación se analiza el parámetro de información de diagnóstico PID's del sensor de temperatura del refrigerante del motor (ECT) del sistema de control electrónico de inyección multipunto FORD EFI. La recolección de información del funcionamiento del sensor de temperatura es obtenida mediante la utilización del escáner automotriz que a través de su conexión a través de la línea de datos obtiene la temperatura del motor de combustión interna en tiempo real, es necesario obtener los valores de la resistencia del termistor a diferentes temperaturas. Para el análisis de datos se aplica la regresión del tipo no lineal exponencial, la cual determina la relación entre variables del tipo dependientes y no dependientes que no están relacionadas de forma lineal, para de esta forma obtener la ecuación base para generar la curva que representa el funcionamiento del sensor de temperatura del motor (ECT). La curva obtenida representa es de gran utilidad en el diagnóstico de sistemas electrónicos y análisis del comportamiento de este tipo de sensores usados en los automóviles.

Palabras claves: diagnóstico electrónico; sensor de temperatura; regresión; inyección electrónica; ect; efi; metodología

Abstract: Through the experimentation in this research the PID diagnostic parameter of the engine coolant temperature sensor (ECT) of the FORD EFI electronic multipoint injection control system is analyzed. The collection of information on the operation of the temperature sensor is obtained by using specialized automotive instrumentation that through its connection through the data line obtains the temperature of the internal combustion engine in real time, it is necessary to obtain the values of the resistance of the thermistor to different temperatures. For the data analysis, the regression of the exponential nonlinear type is applied, which determines the relationship between dependent and nondependent type variables that are not linearly related, in order to obtain the base 
equation to generate the curve that represents the operation of the engine temperature sensor (ECT). The obtained curve represents is very useful in the diagnosis of electronic systems and behavioral analysis of this type of sensors used in cars.

Key words: electronic diagnosis; temperature sensor; nonlinear regression; electronic injection; ect; efi; methodology

\section{Introducción}

La evolución en los sistemas de inyección electrónica de combustible le permite al motor de combustión interna trabajar de manera eficiente el consumo de combustible que ingresa para ser combustionada, modificar los parámetros de funcionamiento y de manera inteligente reducir las emisiones sin tener modificaciones en el rendimiento y la potencia del motor de combustión interna.

Quiroz J \& Quiroz L. (2005) mencionan que: La unidad de electrónica (ECU) controla, analiza, comanda la información de sensores y actuadores, otra función de la computadora automotriz es la de controlar el tiempo que permanecen abiertos los inyectores para optimizar los parámetros característicos del motor de combustión interna.

El análisis del funcionamiento de los sensores y actuadores implementados en vehículos son de interés, Erazo G. (2013) es un campo muy amplio de estudio, para el cual es necesario utilizar equipo de diagnóstico especializado que obtienen valores y curvas de funcionamiento de cualquier componente eléctrico o electrónico del sistema de inyección electrónica de combustible, estas curvas y valores le permiten al especialista determinar si el valor arrojado por el sensor es el correcto.

Al utilizar un modelo matemático como la regresión, Bowerman et Al. (2007) se obtienen la curva teórica de funcionamiento, la cual se utiliza para compararla con la curva experimental del sensor que se está investigando, de esta manera se inserta el método de análisis mediante el estudio y la comparación de dos curvas de tendencia de funcionamiento.

El motor con inyección electrónica dispone de varios sensores, entre ellos el de temperatura de refrigerante del motor (ECT) que determina la temperatura del mismo a través de la variación de la resistencia y voltaje, a la unidad de control electrónico Laverde C. \& Panchi D. (2007) efectúan múltiples acciones como: controlar la inyección de combustible, ajustar el tiempo de encendido, efectuar la sincronización variable de válvulas, activar los cambios de trasmisión. Entre otras acciones que mejoran rendimiento del motor Cerda D. \& Pazmiño I. (2011).

El sensor de temperatura del refrigerante del motor ECT está en contacto con el fluido en refrigerante en el cabezote, el bloque o en el colector de admisión. Es un pasador roscado hueco que tiene una resistencia sellada en su interior Vangelder K. (2010).

Los sensores de temperatura ECT son termistores de coeficiente negativo de dos terminales. Un terminal se conecta a tierra de la ECU, el otro al voltaje de referencia (5.00 voltios). Cuando la temperatura se eleva la resistencia baja y también baja el voltaje a través del 
ECT. Mientras más alta la temperatura, más bajo el voltaje a través del sensor ECT según Pei, W. et Al. (2014).

La mayoría de los sensores de temperatura son termistores del tipo NTC (Coeficiente de Temperatura Negativo). Es decir, la resistencia interna del sensor irá disminuyendo con el incremento de la temperatura medida. Cuando está frio el sensor su conductibilidad es mala y esta aumenta con el incremento de la temperatura. Fabre, L., \& Fonzes, G. (2007)

El sensor ECT al ser un termistor del tipo negativo NTC, obtiene su variación de resistencia mediante el cambio de temperatura del refrigerante. Cuando la temperatura del refrigerante del motor es baja $\left(20^{\circ} \mathrm{C}\right)$ la resistencia del termistor es de $2.5 \mathrm{k} \square$, al contrario, cuando la temperatura del refrigerante del motor es alta $\left(80^{\circ} \mathrm{C}\right)$ la resistencia del termistor es de $0,05 \mathrm{k} \square$ y se usa como señal de instrumentación en el panel de instrumentos de acuerdo a Ruettiger, A. (2008).

Otra relación en el sensor de temperatura del refrigerante (ECT) es la que existe entre el voltaje de señal y el cambio de temperatura. El motor a temperatura de funcionamiento esta en 0.65 volts. Las lecturas del motor de combustión en frío varían dependiendo de la temperatura entre 3.5 voltios a $10^{\circ} \mathrm{C}$ y 2.5 volts a $35^{\circ} \mathrm{C}$, Santander, J. (2005).

La regresión lineal no siempre da buenos resultados, porque a veces la relación entre Y y $\mathrm{X}$ no es lineal, sino que exhibe algún grado de curvatura Baty, F., et Al. (2015). Se entiende por regresión no lineal cuando las variables X e Y están relacionadas de cualquier manera excepto de forma lineal.

\section{Método}

La investigación se desarrolla considera los parámetros de información de diagnóstico obtenidos del sistema de control de inyección electrónica multipunto EFI de la marca FORD, así como el uso de equipos de tecnología de punta como osciloscopio, multímetro, escáner y software de aplicación, por lo tanto, se amplía los resultados a través del método de matematización para todos los motores con inyección electrónica ya que estos disponen como base el sensor de temperatura del refrigerante ECT, el esquema metodológico se presenta en la figura 1.

El método deductivo se enfoca en el análisis de los parámetros de información de diagnóstico que presenta, el análisis que se realiza para construir curvas que se enfoquen en el funcionamiento idóneo del sistema, para determinar el estado de los componentes mediante la curva resultante del proceso.

El método analítico analiza y clasifica la información para su posterior empleo en relaciones y cálculos matemáticos necesarios para la obtención de las curvas.

Al utilizar el método de síntesis mediante las funciones de las curvas del funcionamiento idóneo de los componentes de la inyección electrónica multipunto se generaliza acerca no solo del proceso estadístico de cálculo para la obtención de dichas curvas para todos los sistemas que 
disponen de datos de información en su sistema electrónico, sino también de una idea más concreta de su funcionamiento idóneo.

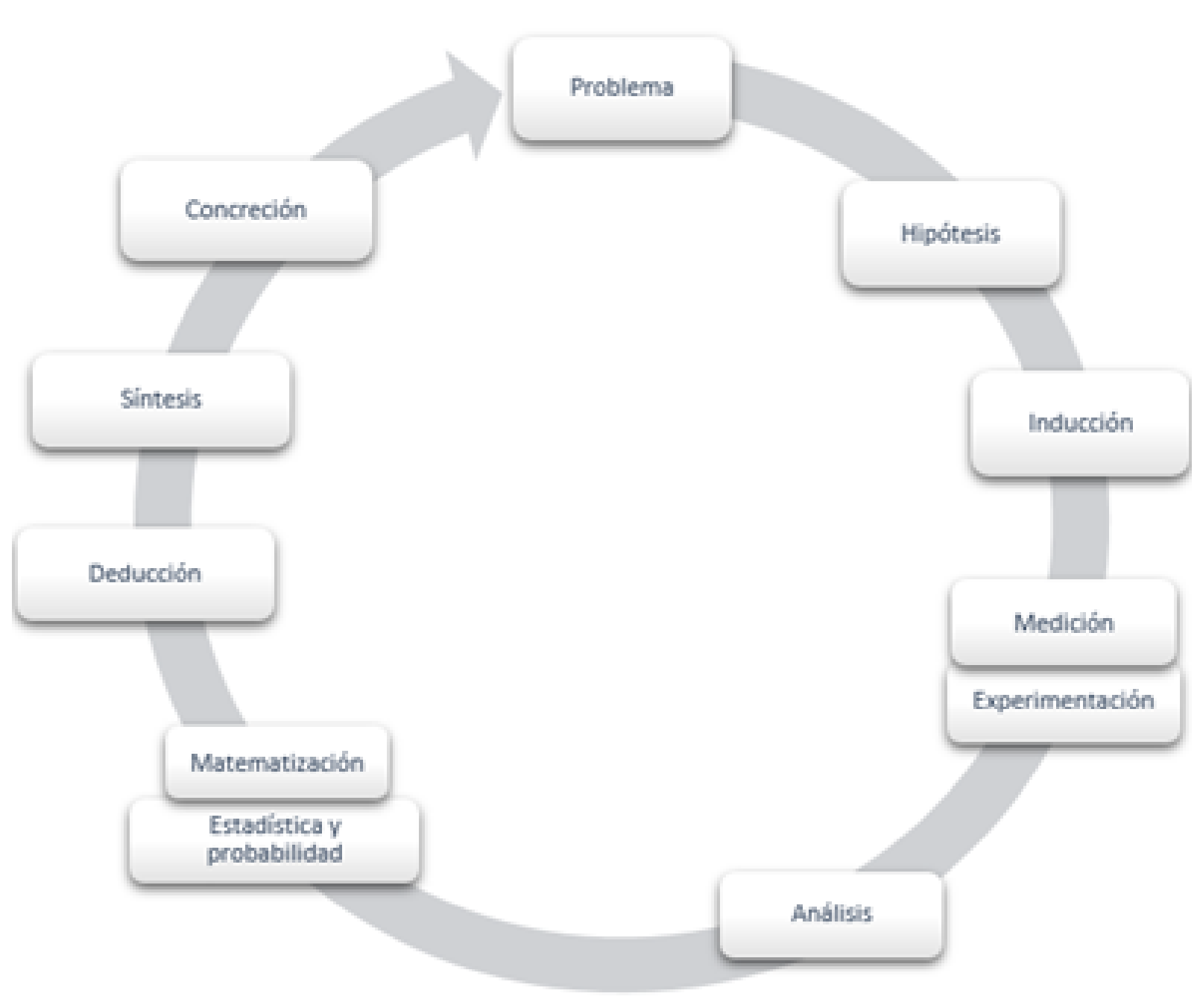

Figura 1. Metodología utiliza en la investigación del sistema matemático de regresión del sensor de temperatura del líquido refrigerante

El método experimental se utiliza para a través de equipos de medición obtener los parámetros de información de diagnóstico para controlar distintas condiciones de operación del motor de combustión interna. Considerando la toma de las variables, referidos a los parámetros de identificación de diagnóstico de los sensores y actuadores del sistema de control electrónico multipunto EFI, mediante el empleo de un protocolo de pruebas.

El método de matematización utiliza el modelo matemático de regresión para la formación de la curva de tendencia con su respectiva ecuación, misma que ejemplifica el funcionamiento ideal del sistema de control electrónico de gasolina multipunto EFI. 
A través del método de la concreción se obtienen los valores resultantes y las ecuaciones correspondientes para ser aplicados en cualquier sistema de control de temperatura del motor de combustión interna.

Se conecta el escáner de diagnóstico al DLC (data link connector) y se procede a observar las variaciones de temperatura, cuando la temperatura llegue a la esperada se mide la resistencia del termistor y se llena la tabla 1 , con 5 muestras.

Establecida la metodología con el uso del escáner que es conectado a la línea de datos DLC se obtienen cinco mediciones de desempeño del sensor de temperatura del motor considerando valores de 20 a 100 grados centígrados de temperatura utilizando refrigerante debidamente aditivado lo que genera la tabla 1.

Tabla 1. Datos del sensor de temperatura del refrigerante del motor obtenidos a diferentes temperaturas

\begin{tabular}{ccc}
\hline $\mathbf{N}^{\mathbf{0}}$ & Temperatura $\left({ }^{\circ} \mathbf{C}\right)$ & Resistencia del termistor $(\mathbf{k} \boldsymbol{\Omega})$ \\
\hline $\mathbf{1}$ & 20 & 3.520 \\
$\mathbf{2}$ & 40 & 1.459 \\
$\mathbf{3}$ & 60 & 0.667 \\
$\mathbf{4}$ & 80 & 0.332 \\
$\mathbf{5}$ & 100 & 0.177 \\
\hline
\end{tabular}

El diagrama de dispersión utiliza los datos obtenidos en la experimentación del sensor de temperatura que se encuentran se registran en tabla 1. Se utiliza el software graficador GRAPH para disponer de la designación de puntos en el plano cartesiano, en donde la temperatura del refrigerante se encontrará en el eje de las abscisas y la resistencia del termistor en el eje de las ordenadas. 


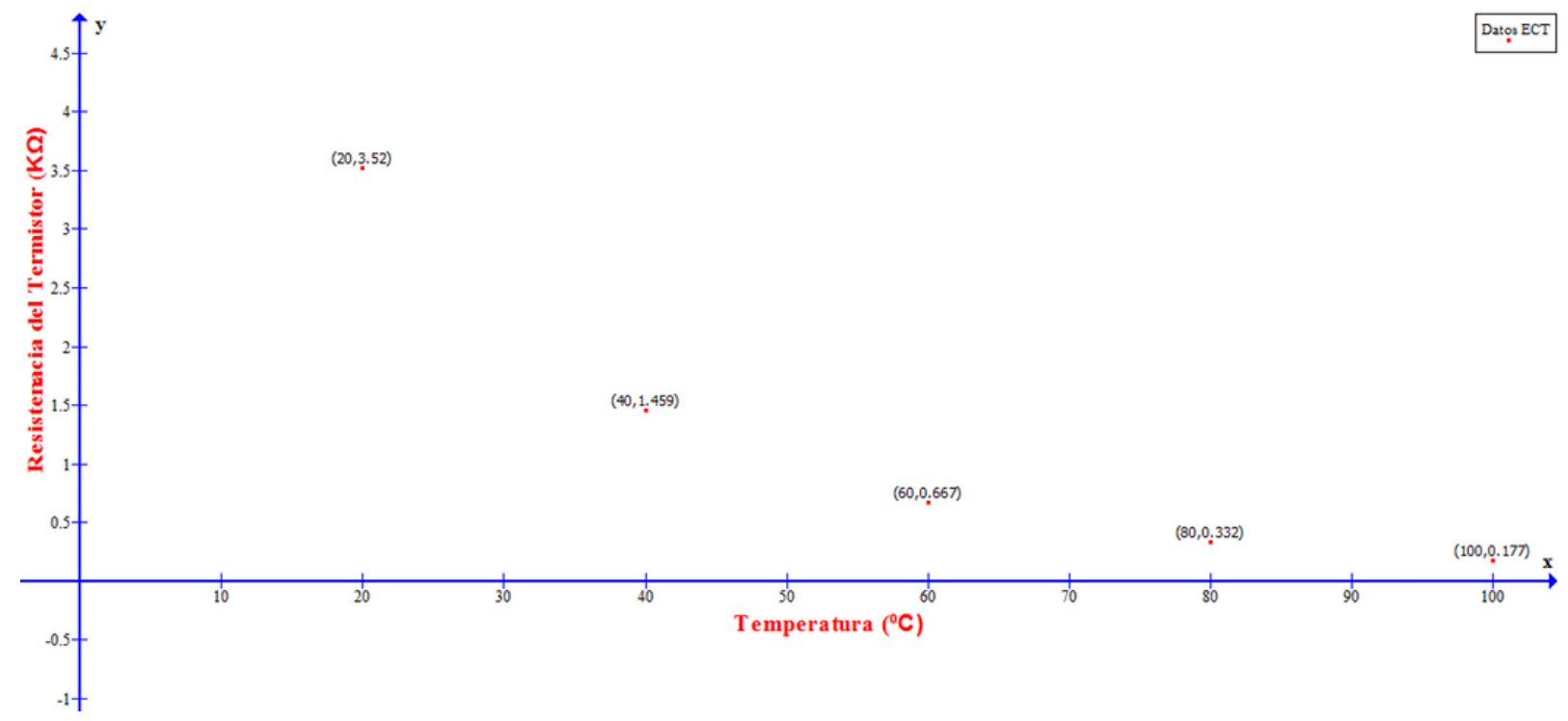

Figura 2. Diagrama de dispersión obtenidos en el proceso de medición del sensor de temperatura

En la obtención de la curva teórica de funcionamiento del sensor de temperatura ECT, se utiliza la regresión no lineal por el método exponencial. Archontoulis, S. V., \& Miguez, F. E. (2015). La regresión exponencial es el proceso para encontrar la ecuación de la función exponencial que se ajuste mejor a un conjunto de datos, para su obtención se utiliza la siguiente expresión:

$$
y=\alpha \cdot \beta^{x} \text { Ecuación } 1
$$

De donde:

$\alpha$ y $\beta=$ estimaciones de los parámetros conocidos de la curva.

Se considera los logaritmos en ambos miembros:

$$
\log y=\log \alpha+x \log \beta \quad \text { Ecuación } 2
$$

Ahora se puede estimar $\log \alpha$ y $\log \beta$ para a continuación encontrar $\alpha$ y $\beta$, aplicando el método de mínimos cuadrados, las constantes quedan fijadas al resolver simultáneamente las ecuaciones.

$$
\begin{array}{ll}
\sum \log y=\log \alpha \cdot N+\log \beta \cdot \sum x & \text { Ecuación 3 } \\
\sum x \cdot \log y=\log \alpha \cdot \sum x+\log \beta \cdot \sum x^{2} & \text { Ecuación } 4
\end{array}
$$

De donde:

$$
N=\text { (Número de datos del sensor) }
$$


Luego de resolver las ecuaciones se reemplaza los valores de y en la ecuación 1. Para de esta manera encontrar la ecuación base que determina la curva de funcionamiento teórica del sensor ECT.

\section{Resultados}

Con los datos recolectados en la tabla 1, se procede a completar los datos requeridos en el análisis de regresión, teniendo en cuenta que los valores de Xi pertenecen a los valores de temperatura del refrigerante del motor y los valores de Yi son los valores de la resistencia del termistor, obteniendo los siguientes resultados.

Tabla 2. Datos de temperatura y voltaje del sensor de temperatura ECT necesarios para la resolución de las ecuaciones

\begin{tabular}{cccccc}
\hline Medición & $\boldsymbol{X}$ & $\boldsymbol{Y}$ & $\boldsymbol{\operatorname { l o g } Y}$ & $\boldsymbol{X}^{2}$ & $\boldsymbol{X} \log \boldsymbol{\operatorname { l o g }}$ \\
\hline $\mathbf{1}$ & 20 & 3.52 & 0.547 & 400 & 10.94 \\
$\mathbf{2}$ & 40 & 1.459 & 0.164 & 1600 & 6.56 \\
$\mathbf{3}$ & 60 & 0.667 & -0176 & 3600 & -10.56 \\
$\mathbf{4}$ & 80 & 0.332 & -0.479 & 6400 & -38.32 \\
$\mathbf{5}$ & 100 & 0.177 & -0.752 & 10000 & -75.2 \\
\hline
\end{tabular}

Tabla 3. Datos de sumatorias de voltaje y temperatura calculados requeridos para la resolución de las ecuaciones

\begin{tabular}{ccc}
\hline Orden & Sumatoria & Resultado \\
\hline $\mathbf{1}$ & $\sum X=$ & 300 \\
$\mathbf{3}$ & $\sum \log Y=$ & -0.696 \\
4 & $\sum X^{2}=$ & 22000 \\
& $\sum X \cdot \log Y=$ & -106.58
\end{tabular}

Se reemplaza los valores obtenidos en la tabla 3 en las ecuaciones que permiten obtener el modelo base. 


$$
\begin{gathered}
\left\{\begin{array}{c}
\sum \log y=\log \alpha \cdot N+\log \beta \cdot \sum x \\
\sum x \cdot \log y=\log \alpha \cdot \sum x+\log \beta \cdot \sum x^{2}
\end{array}\right. \\
\left\{\begin{array}{c}
-0.696=\log \alpha \cdot 5+\log \beta \cdot 300 \\
-106.58=\log \alpha \cdot 300+\log \beta \cdot 22000
\end{array}\right. \\
\left\{\begin{array}{c}
5 \cdot \log \alpha+300 \cdot \log \beta=-0.696 \\
300 \cdot \log \alpha+22000 \cdot \log \beta=-106.58
\end{array}\right.
\end{gathered}
$$

Se obtienen dos ecuaciones con dos incógnitas, se aplica el método de resolución de ecuaciones por matrices para resolver el sistema de ecuaciones hasta obtener el valor de.

$$
\begin{gathered}
\log \alpha=\frac{\Delta \alpha}{\Delta}=\frac{\left|\begin{array}{cc}
-0.696 & 300 \\
-106.58 & 22000
\end{array}\right|}{\left|\begin{array}{cc}
5 & 300 \\
300 & 22000
\end{array}\right|} \\
\log \alpha=\frac{-15312-(-31974)}{110000-90000} \\
\log \alpha=\frac{16662}{20000} \\
\log \alpha=0.8331 \\
\alpha=6.809
\end{gathered}
$$

Una vez encontrado el valor de se procede a determinar el valor de $\beta$.

$$
\begin{gathered}
\log \beta=\frac{\Delta \beta}{\Delta}=\frac{\left|\begin{array}{cc}
5 & -0.696 \\
300 & -106.58
\end{array}\right|}{\left|\begin{array}{cc}
5 & 300 \\
300 & 22000
\end{array}\right|} \\
\log \beta=\frac{-532.90-(208.8)}{110000-90000} \\
\log \beta=\frac{-324.10}{20000} \\
\log \beta=-0.016 \\
\beta=0.963
\end{gathered}
$$


De esta manera finaliza la resolución de la ecuación al sustituir los valores de las estimaciones de los parámetros conocidos de la curva en la ecuación 1 de tipo exponencial

$$
\begin{gathered}
y=\alpha \cdot \beta^{x} \\
y=6.809 * 0.963^{x}
\end{gathered}
$$

Al obtener la ecuación base se procede a ingresar la ecuación en el programa GRAPH para la obtención de la curva del tipo exponencial que representa el funcionamiento teórico del sensor ECT.

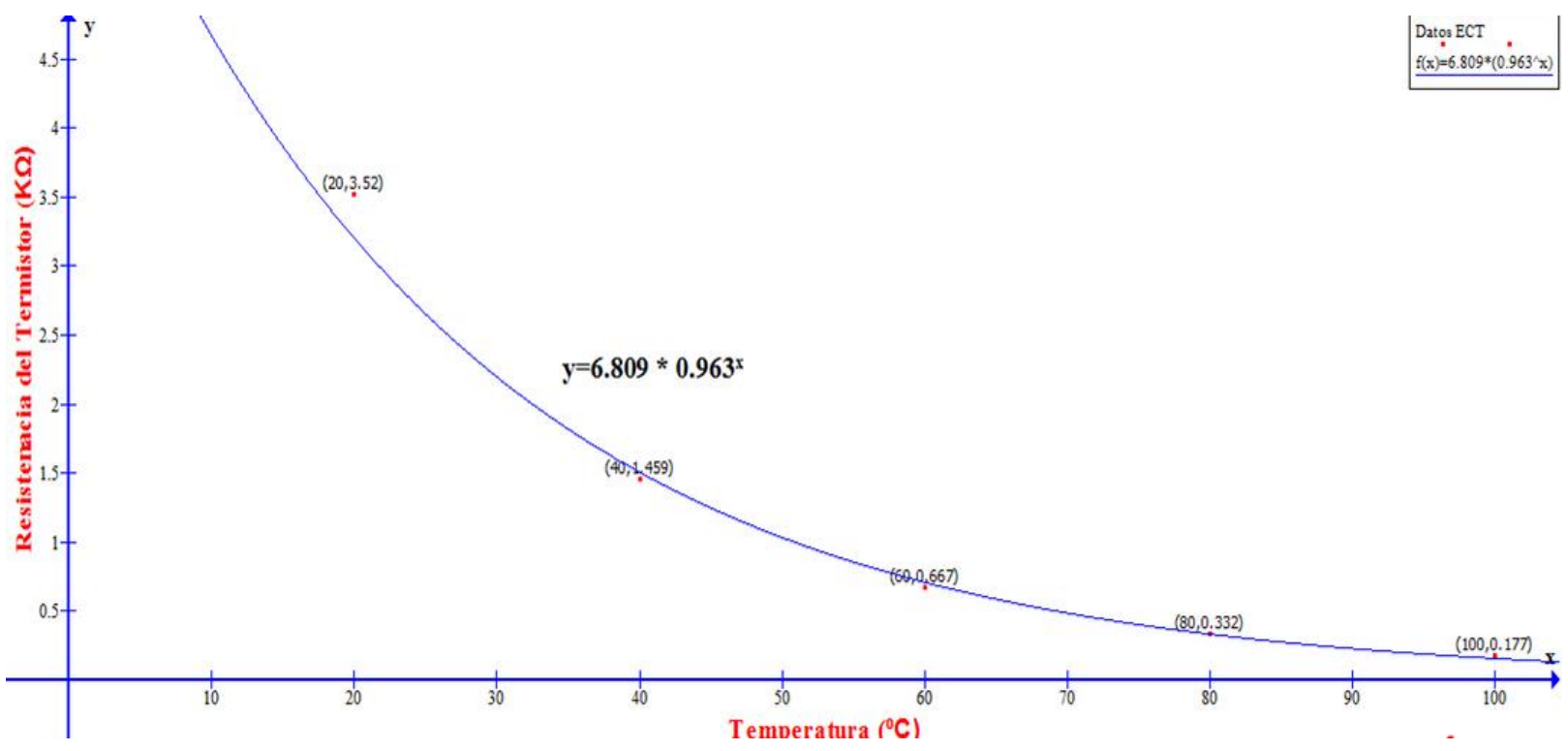

Figura 3. Curva de funcionamiento del sensor de temperatura de refrigerante (ECT)

\section{Conclusiones}

El sensor de temperatura del refrigerante ECT del motor de combustión interna con control de inyección electrónica EFI genera una curva del tipo exponencial la cual está influenciada por los valores de la temperatura del refrigerante y la resistencia del termistor, estos valores tienen una correlación negativa entre sí.

Al generar el diagrama de dispersión se observó cómo los puntos proyectados siguen una tendencia del tipo exponencial que es utilizada por la unidad de control electrónico para ajustar el tiempo de inyección de combustible.

La ecuación base exponencial al ser graficada muestra un modelo teórico del correcto funcionamiento del sensor ECT, el modelo matemático es aplicable en cualquier tipo de sensor térmico. 
La curva generada a partir de la ecuación base no atraviesa necesariamente los puntos de dispersión, ya que la curva proyectada es una curva teórica que se ajusta a los mismos.

La curva proyectada a partir de los datos reales del sensor de temperatura, puede ser utilizada para diagnosticar fallos en el trabajo de cualquier sistema de control del tipo electrónico multipunto, mediante el análisis y la comparación.

Es indispensable desarrollar la matematización a través del sistema de regresión no lineal para argumentar el desempeño de los sensores y actuadores del control electrónico de inyección de gasolina de tal forma que se tenga clara la concepción de como la unidad de control electrónico utilizará estos valores para procesar la información generada en el vehículo.

\section{Bibliografía}

Archontoulis, S. V., \& Miguez, F. E. (2015). Nonlinear regression models and applications in agricultural research. Agronomy Journal, 107(2), 786-798

Baty, F., Ritz, C., Charles, S., Brutsche, M., Flandrois, J. P., \& Delignette-Muller, M. L. (2015). A toolbox for nonlinear regression in R: the package nlstools. Journal of Statistical Software, 66(5), 1-21.

Bosch, R (2015). Inyección electrónica: Fuel Injection. México, México D. F.: Boschautopartes

Bowerman, B. L., Richard, T., II, K., \& Anne, B. (2007). Pronósticos, series de tiempo y regresión: un enfoque aplicado/Bruce L. Bowerman, Richard T O'Connell, Anne B. Koehler (No. 519.232 B6Y 2006.)

Cerda Sánchez, D. A., \& Pazmiño Días, I. P. (2011). Diseño e implementación de un sistema con GPS y control de seguridad vehicular con comunicación GSM (Bachelor's thesis, Latacunga/ESPE/2011).

Erazo Laverde, G., \& López Espinel, E. (2005). Diseño y construcción de un banco de pruebas para limpieza y comprobación de inyectores a gasolina (Bachelor's thesis, Latacunga/ESPE/2005).

Fabre, L., \& Fonzes, G. (2007). U.S. Patent No. 7,209,855. Washington, DC: U.S. Patent and Trademark Office. [4].

Laverde Albarracín, C. S., \& Panchi Mayo, D. O. (2007). Diseño e implementación de un módulo de entrenamiento para inyección electrónica a gasolina del vehículo chevrolet vitara g1600 del laboratorio de motores (Bachelor's thesis, LATACUNGA/ESPE/2007).

Pei, W., Lan, Y., Xiwen, L., Zhiyan, Z., Wang, Z., \& Wang, Y. (2014). Integrated sensor system for monitoring rice growth conditions based on unmanned ground vehicle system. International Journal of Agricultural and Biological Engineering, 7(2), 75. 
Quiroz Erazo, J. L., \& Quiroz Erazo, L. A. (2005). Diseño y construcción de un módulo de entrenamiento del sistema de inyección electrónica de gasolina del vehículo Corsa Wind.

Ruettiger, A. (2008). U.S. Patent No. 7,325,972. Washington, DC: U.S. Patent and Trademark Office.

Santander, J. R (2005). Manual técnico Fuel Injection. Ecuador, Guayaquil: Diseli Editoriales. 